\title{
POWER SERIES WITH INTEGRAL COEFFICIENTS
}

\author{
BY DAVID G. CANTOR
}

Communicated by P. T. Bateman, January 17, 1963

Let $f(z)$ be a function, meromorphic in $|z|<1$, whose power series around the origin has integral coefficients. In [5], Salem shows that if there exists a nonzero polynomial $p(z)$ such that $p(z) f(z)$ is in $H^{2}$, or else if there exists a complex number $\alpha$, such that $1 /(f(z)-\alpha)$ is bounded, when $|z|$ is close to 1 , then $f(z)$ is rational. In [2], Chamfy extends Salem's results by showing that if there exists a complex number $\alpha$ and a nonzero polynomial $p(z)$, such that $p(z) /(f(z)-\alpha)$ is in $H^{2}$, then $f(z)$ is rational. In this paper we show that if $f(z)$ is of bounded characteristic in $|z|<1$ (i.e. the ratio of two functions, each regular and bounded in $|z|<1)$, then $f(z)$ is rational. If $f(z)$ is regular in $|z|<1$, then, by [4], $f(z)$ is of bounded characteristic in $|z|<1$, if and only if

$$
\limsup _{r \rightarrow 1-} \int_{0}^{2 \pi} \log ^{+}\left|f\left(r e^{i \theta}\right)\right| d \theta<\infty .
$$

Thus any function in any $H^{p}$ space $(p>0)$ is of bounded characteristic. Hence, since the functions of bounded characteristic form a field, our result includes those of Salem and Chamfy.

Our first lemma gives a necessary condition for a function to be of bounded characteristic in $|z|<1$, in terms of the properties of its Taylor series coefficients.

If $g(z)=\sum_{i=0}^{\infty} a_{i} z^{i}$, we denote by $A_{r}=A_{r}(g)$ the matrix $\left\|a_{i+j}\right\|$, $0 \leqq i, j \leqq r$.

Lemma 1. Suppose $g(z)$ is of bounded characteristic in $|z|<1$. Then $\operatorname{det}\left(A_{r}\right) \rightarrow 0$ as $r \rightarrow \infty$. More precisely, $\lim _{r \rightarrow \infty}\left|\operatorname{det}\left(A_{r}\right)\right|^{1 / r}=0$.

Proof. By assumption, we may write $g(z)=s(z) / t(z)$, where $s(z)$ and $t(z)$ are bounded analytic functions in $|z|<1$. Suppose that $s(z)=\sum_{i=0}^{\infty} s_{i} z^{i}$ and $t(z)=\sum_{i=0}^{\infty} t_{i} z^{i}$, and, without loss of generality, that $t_{0}=1$. We now perform a series of column and row operations on the matrix $A_{r}$. Denote its columns from left to right by $c_{0}, c_{1}$, $c_{2}, \cdots, c_{r}$. Now, successively, for $j=0,1,2, \cdots, r$, replace the column $c_{r-j}$ by $\sum_{i=0}^{r-j} t_{i} c_{r-j-i}$; then perform the same sequence of operations on the rows. This yields a matrix $D_{r}=\left\|d_{m n}\right\|, 0 \leqq m, n \leqq r$. Since $t_{0}=1, \operatorname{det}\left(D_{r}\right)=\operatorname{det}\left(A_{r}\right)$. 
It is easy to verify that

$$
d_{m n}=\sum_{i=0}^{m} \sum_{j=0}^{n} t_{i} t_{j} a_{m+n-i-j} .
$$

Hence $d_{m n}$ is the coefficient of $z^{m+n}$ in

$$
\begin{aligned}
\sum_{i=0}^{m} t_{i} z^{i} \sum_{j=0}^{n} t_{j} z^{j} \sum_{k=0}^{\infty} a_{k} z^{k} & \left(t(z)-\sum_{i=m+1}^{\infty} t_{i} z^{i}\right)\left(t(z)-\sum_{j=n+1}^{\infty} t_{j} z^{j}\right) g(z) \\
= & \left(t(z)-\sum_{i=m+1}^{\infty} t_{i} z^{i}-\sum_{j=n+1}^{\infty} t_{j} z^{j}\right) s(z)+g(z) \sum_{i=m+1}^{\infty} t_{i} z^{i} \sum_{j=n+1}^{\infty} t_{j} z^{j}
\end{aligned}
$$

since $t(z) g(z)=s(z)$. As the coefficient of $z^{m+n}$ in the last term of (1) is $0, d_{m n}$ is the coefficient of $z^{m+n}$ in

Hence

$$
\left(\sum_{j=0}^{m} t_{j} z^{j}+\sum_{j=0}^{n} t_{j} z^{j}-\sum_{j=0}^{\infty} t_{j} z^{j}\right) \sum_{j=0}^{\infty} s_{j} z^{j} .
$$

$$
d_{m n}=\alpha_{m n}+\alpha_{n m}-\beta_{m n}
$$

where $\alpha_{m n}=\sum_{i=0}^{m} t_{i} s_{m+n-i}$ and $\beta_{m n}=\sum_{i=0}^{m+n} t_{i} s_{m+n-i}$. Then, by Schwarz's inequality,

$$
\left|d_{m n}\right|^{2} \leqq 3\left(\left|\alpha_{m n}\right|^{2}+\left|\alpha_{n m}\right|^{2}+\left|\beta_{m n}\right|^{2}\right)
$$

We now show that

$$
\sum_{m=0}^{r} \sum_{n=0}^{r}\left|d_{m n}\right|^{2}=o(r)
$$

To do this, it suffices to show that

and that

$$
\sum_{m=0}^{r} \sum_{n=0}^{r}\left|\alpha_{m n}\right|^{2}=o(r)
$$

$$
\sum_{m=0}^{r} \sum_{n=0}^{r}\left|\beta_{m n}\right|^{2}=o(r) .
$$

Now, $\alpha_{m n}$ is the coefficient of $z^{m+n}$ in $\sum_{i=0}^{\infty} t_{i} z^{i} \sum_{j=n}^{\infty} s_{j} z^{i}$. Hence, by Parseval's equality,

$$
\sum_{m=0}^{\infty}\left|\alpha_{m n}\right|^{2}=\lim _{\rho \rightarrow 1-} \frac{1}{2 \pi} \int_{0}^{2 \pi}\left|t(z) \sum_{i=n}^{\infty} s_{i} z^{i}\right|^{2} d \theta,
$$


where $z=\rho e^{i \theta}$. Now $t(z)$ is bounded in $|z|<1$ by, say, $T$. Thus, again using Parseval's equality, we have, when $|z|=\rho<1$,

$$
\begin{aligned}
\frac{1}{2 \pi} \int_{0}^{2 \pi}\left|t(z) \sum_{i=n}^{\infty} s_{i} z^{i}\right|^{2} d \theta & \leqq \frac{T^{2}}{2 \pi} \int_{0}^{2 \pi}\left|\sum_{i=n}^{\infty} s_{i} z^{i}\right|^{2} d \theta \\
& =T^{2} \sum_{i=n}^{\infty}\left|s_{i}\right|^{2} \rho^{2 i} .
\end{aligned}
$$

Put $S_{n}=\sum_{i=n}^{\infty}\left|s_{i}\right|^{2}$. Then, as $s(z)$ is bounded, $S_{0}$ is finite and $S_{n} \rightarrow 0$ as $n \rightarrow \infty$. By (3) and (4), we have

$$
\sum_{m=0}^{\infty}\left|\alpha_{m n}\right|^{2} \leqq T^{2} S_{n}
$$

Hence

$$
\sum_{n=0}^{r} \sum_{m=0}^{r}\left|\alpha_{m n}\right|^{2} \leqq T^{2} \sum_{n=0}^{r} S_{n}=o(r) .
$$

Now, $\beta_{m n}$ is the coefficient of $z^{m+n}$ in the bounded function $s(z) t(z)$ $=\sum_{i=0}^{\infty} u_{i} z^{i}$. Then,

$$
\sum_{m=0}^{r}\left|\beta_{m n}\right|^{2} \leqq \sum_{m=0}^{\infty}\left|\beta_{m n}\right|^{2}=\sum_{i=n}^{\infty}\left|u_{i}\right|^{2} .
$$

Thus,

$$
\sum_{n=0}^{r} \sum_{m=0}^{r}\left|\beta_{m n}\right|^{2}=o(r)
$$

Hence, by (2), (5), and (6),

$$
\sum_{m=0}^{r} \sum_{n=0}^{r}\left|d_{m n}\right|^{2}=o(r) .
$$

We now estimate $\operatorname{det}\left(D_{r}\right)$. By Hadamard's inequality,

$$
\left|\operatorname{det}\left(D_{r}\right)\right|^{2} \leqq \prod_{m=0}^{r} \sum_{n=0}^{r}\left|d_{m n}\right|^{2} .
$$

The right hand side of $(7)$ is the $(r+1)$ st power of the geometric mean of the quantities $\sum_{n=0}^{r}\left|d_{m n}\right|^{2}, 0 \leqq m \leqq r$. Hence, by the inequality between arithmetic and geometric means

$$
\left|\operatorname{det}\left(D_{r}\right)\right|^{2 /(r+1)} \leqq \frac{1}{r+1} \sum_{m=0}^{r} \sum_{n=0}^{r}\left|d_{m n}\right|^{2}=o(1) .
$$


Hence, since $\operatorname{det}\left(D_{r}\right)=\operatorname{det}\left(A_{r}\right)$, we have

$$
\lim _{r \rightarrow \infty}\left|\operatorname{det}\left(A_{r}\right)\right|^{1 / r}=0 .
$$

By a change of variable we obtain

Lemma 2. Suppose $g(z)$ is regular at $z=0$, and of bounded characteristic in $|z|<s$. Then $\lim _{r \rightarrow \infty} s^{r}\left|\operatorname{det}\left(A_{r}(g)\right)\right|^{2 / r}=0$.

THEOREM 1. Let $f(z)$ be a function of bounded characteristic in $|z|<1$, whose Laurent series around the origin has integral coefficients. Then $f(z)$ is rational.

Proof. By multiplying $f(z)$ by a power of $z$, if necessary, we may assume that $f(z)$ is regular at $z=0$, and has a power series expansion $f(z)=\sum_{i=0}^{\infty} a_{i} z^{i}$, where the $a_{i}$ are integers. By Lemma 1, $\lim _{n \rightarrow \infty} \operatorname{det}\left(A_{n}(f)\right)=0$. As the $a_{i}$ are integers, so are the $\operatorname{det}\left(A_{n}(f)\right)$. It follows that $\operatorname{det}\left(A_{n}(f)\right)=0$ for all large $n$. But this implies that $f(z)$ is rational, by a theorem by Kronecker [1, p. 138].

Corollary. Let $f(z)$ be a function meromorphic in $|z|<1$, whose Laurent series around the origin has integral coefficients. If there exists a set $S$ of positive capacity, such that for each $\alpha \in S$, the equation $f(z)=\alpha$ has only finitely many solutions in $|z|<1$, then $f(z)$ is rational.

Proof. If $f(z)$ satisfies only the second condition, then by a theorem of Frostman [3] or [4, p. 260], $f(z)$ is of bounded characteristic. q.e.d.

Let $K$ be an algebraic number field of degree $n$ over the rationals; denote by $K^{(i)}, 1 \leqq i \leqq n$, the different embeddings of $K$ into the field of complex numbers. If $a \in K$, denote by $a^{(i)}$ the image of $a$ in $K^{(i)}$.

THEOREM 2. Let $f(z)=\sum_{j=0}^{\infty} a_{j} z^{j}$ be a formal power series whose coefficients $a_{j}$ are algebraic integers in $K$. Suppose that $f^{(i)}(z)=\sum_{j=0}^{\infty} a_{j}^{(i)} z^{j}$ is of bounded characteristic in the disc $|z|<s_{i}, 1 \leqq i \leqq n$, where $\prod_{i=1}^{n} s_{i}$ $\geqq 1$. Then $f(z)$ is a rational function.

Proof. Put $A_{r}=A_{r}(f)$ and $A_{r}^{(i)}=A_{r}\left(f^{(i)}\right)$. By Lemma 2, $s_{i}^{r}\left|\operatorname{det} A_{r}^{(i)}\right|{ }^{2 / r} \rightarrow 0$ as $r \rightarrow \infty$. Hence

$$
\mathrm{Nm} \operatorname{det}\left(A_{r}\right)=\prod_{i=1}^{n} \operatorname{det}\left(A_{r}^{(i)}\right) \rightarrow 0
$$

as $r \rightarrow \infty$. Since $\mathrm{Nm} \operatorname{det}\left(A_{r}\right)$ is an integer, it is eventually 0 . Hence by the theorem of Kronecker (whose proof is valid over any field) [1, p. 138], $f(z)$ is rational. 


\title{
REFERENCES
}

1. J. Cassels, An introduction to Diophantine approximation, Cambridge Tract 45, Cambridge Univ. Press, Cambridge, 1957.

2. C. Chamfy, Fonctions méromorphes dans le cercle-unité et leurs series de Taylor, Ann. Inst. Fourier Grenoble 8 (1958), 237-245.

3. O. Frostman, Über die defecten Werte einer meromorphen Funktion, 8 Congr. Math. Scand., Stockholm, 1934. 1936.

4. R. Nevanlinna, Eindeutige Analytische Funktionen, Springer Verlag, Berlin,

5. R. Salem, Power series with integral coefficients, Duke Math. J. 12 (1945), 153172.

UNIVERSITY OF WASHINGTON

\section{COMMUTING VECTOR FIELDS ON 2-MANIFOLDS}

\author{
BY ELON L. LIMA ${ }^{1}$
}

Communicated by Deane Montgomery, January 28, 1963

We shall consider $C^{1}$ vector fields $X, Y$ on a compact 2-manifold $M$. When the Lie bracket $[X, Y]$ vanishes identically on $M$, we say that $X$ and $Y$ commute. It was shown in [1] that every pair of commuting vector fields on the 2 -sphere $S^{2}$ has a common singularity. Here we extend this result to all compact 2-manifolds with nonvanishing Euler characteristic.

Our manifolds are connected and may have boundary. The boundary of a compact 2-manifold is either empty or consists of finitely many disjoint circles. Given a $C^{1}$ vector field $X$ on a compact manifold $M$, we tacitly assume that $X$ is tangent to the boundary of $M$ (if it exists). Then the trajectories of $X$ are defined for all values of the parameter, and translation along them provides a (differentiable) action $\xi$ of the additive group $R$ on $M$. Given $x \in M$, one has $X(x)=0$ if, and only if, $x$ is a fixed point of $\xi$, that is, $\xi(s, x)=x$ for all $s \in R$. Let $Y$ be another $C^{1}$ vector field on $M$, generating the action $\eta$ of $R$ on $M$. The condition $[X, Y] \equiv 0$ means that $\xi$ and $\eta$ commute, that is, $\xi(s, \eta(t, x))=\eta(t, \xi(s, x))$ for all $x \in M$ and $s, t \in R$. Thus the pair $X, Y$ generates an action $\phi: R^{2} \times M \rightarrow M$ of the additive group $R^{2}$ on $M$, defined by $\phi(r, x)=\xi(s, \eta(t, x))=\eta(t, \xi(s, x))$ for $x \in M$ and $r=(s, t) \in R^{2}$. Notice that $x \in M$ is a fixed point of $\phi$ if, and only if, $x$ is a common singularity of $X$ and $Y$, that is, $X(x)=Y(x)=0$. These

${ }^{1}$ Fellow of the John Simon Guggenheim Memorial Foundation. Work partially supported by the National Science Foundation, under NSF-G21514. 